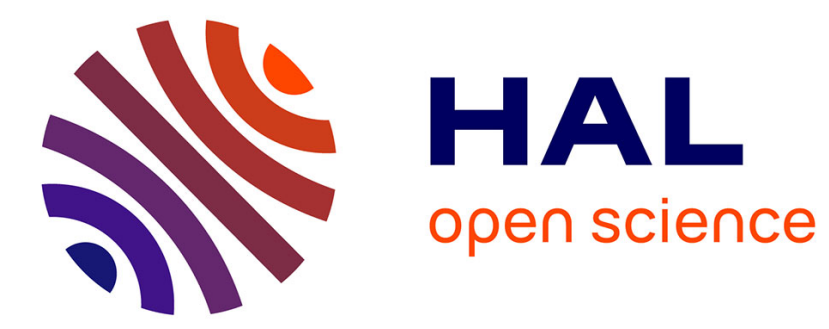

\title{
POROSITY IN THE SPACE OF HÖLDER-FUNCTIONS
}

\author{
Mohammed Bachir
}

\section{To cite this version:}

Mohammed Bachir. POROSITY IN THE SPACE OF HÖLDER-FUNCTIONS. 2021. hal$03222485 \mathrm{v} 1$

\section{HAL Id: hal-03222485}

\section{https://hal-paris1.archives-ouvertes.fr/hal-03222485v1}

Preprint submitted on 10 May 2021 (v1), last revised 30 May 2021 (v2)

HAL is a multi-disciplinary open access archive for the deposit and dissemination of scientific research documents, whether they are published or not. The documents may come from teaching and research institutions in France or abroad, or from public or private research centers.
L'archive ouverte pluridisciplinaire HAL, est destinée au dépôt et à la diffusion de documents scientifiques de niveau recherche, publiés ou non, émanant des établissements d'enseignement et de recherche français ou étrangers, des laboratoires publics ou privés. 


\title{
POROSITY IN THE SPACE OF HÖLDER-FUNCTIONS.
}

\author{
MOHAMMED BACHIR
}

\begin{abstract}
Let $(X, d)$ be a bounded metric space with a base point $0_{X}$, $(Y,\|\cdot\|)$ be a Banach space and $\operatorname{Lip}_{0}^{\alpha}(X, Y)$ be the space of all $\alpha$-Hölderfunctions that vanish at $0_{X}$, equipped with its natural norm $(0<\alpha \leq$ $1)$. Let $0<\alpha<\beta \leq 1$. We prove that $\operatorname{Lip}_{0}^{\beta}(X, Y)$ is a $\sigma$-porous subset of $\operatorname{Lip}_{0}^{\alpha}(X, Y)$, if (and only if) $\inf \left\{d\left(x, x^{\prime}\right): x, x^{\prime} \in X ; x \neq x^{\prime}\right\}=0$ (i.e. $d$ is non-uniformly discrete). A more general result will be given.
\end{abstract}

2010 Mathematics Subject Classification: 26A16; 54E52; 47L05; 46B25 Keyword, phrase: vector-valued Lipschitz and Hölder-functions, vectorvalued Linear operators, $\sigma$-porosity, barrier cone.

\section{INTRODUCTION}

The main result of this note is Theorem 1, which gives a condition for some class of subsets of Lipschitz functions to be $\sigma$-porous subsets. The result in the abstract, as well as all the other results of this note, are just a very immediate consequence of this main result. However, the main motivations which led to the main theorem of this note, was precisely the result mentioned in the abstract.

Given a metric space $(X, d)$ with a distinguished point $0_{X}$ (called a base point of $X$ ) and a Banach space $(Y,\|\cdot\|)$, we denote by $\operatorname{Lip}_{0}\left(X_{d}, Y\right)$ (or by $\operatorname{Lip}_{0}(X, Y)$, if no ambiguity arises) the Banach space of all Lipschitz functions from $X$ into $Y$ that vanish at the base point $0_{X}$, equipped with its natural norm defined by

$$
\|f\|_{L}:=\sup \left\{\frac{\left\|f(x)-f\left(x^{\prime}\right)\right\|}{d\left(x, x^{\prime}\right)}: x, x^{\prime} \in X ; x \neq x\right\}, \forall f \in \operatorname{Lip}_{0}\left(X_{d}, Y\right) .
$$

We denote simply $\operatorname{Lip}_{0}\left(X_{d}\right)$ or $\operatorname{Lip}_{0}(X)$, if $Y=\mathbb{R}$. The space $L(X, Y)$ denotes the space of all linear bounded operators from $X$ into $Y$. The space $X^{*}$

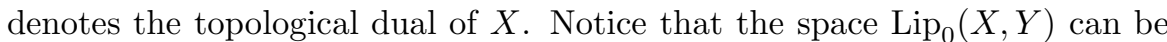
isometrically itentified to $L(\mathcal{F}(X), Y)$ where $\mathcal{F}(X)$ is the free-Lipschitz space over $X$ introduced by Godefroy-Kalton in [2]. Let us recall the definition of $\sigma$-porosity. 
Definition 1. Let $(F, d)$ be a metric space and $A$ be a subset of $F$. A set $A$ of $F$ is called porous if there is a $c \in(0,1)$ so that for every $x \in A$ there are $\left(y_{n}\right) \subset F$ with $y_{n} \rightarrow x$ and so that $B\left(y_{n}, c d\left(y_{n}, x\right)\right) \cap A=\emptyset$ for every $n$ (We denote by $B(z, r)$ the closed ball with center $z$ and radius $r)$. A set $A$ is called $\sigma$-porous if it can be represented as a union $A=\cup_{n=0}^{+\infty} A_{n}$ of countably many porous sets (the porosity constant $c_{n}$ may vary with $n$ ).

Every $\sigma$-porous set is of first Baire category. Moreover, in $\mathbb{R}^{n}$, every $\sigma$ porous set is of Lebesque measure zero. However, there does exist a non- $\sigma$ porous subset of $\mathbb{R}^{n}$ which is of the first category and of Lebesgue measure zero (for more informations about $\sigma$-porosity, we refer to [7] and [5]).

The property $(\mathcal{P})$. Let $(X, d)$ be a metric space and $Y$ be a Banach space. Let $F$ be a nonempty (closed) convex cone of $\operatorname{Lip}_{0}(X, Y)$. We say that $F$ satisfies property $(\mathcal{P})$ if there exists a positive constant $K_{F}>0$ depending only on $F$ such that:

$(\mathcal{P}) \quad \forall\left(x, x^{\prime}\right) \in X \times X, \exists p \in F:\|p\|_{L} \leq K_{F}$ and $\left\|p(x)-p\left(x^{\prime}\right)\right\|=d\left(x, x^{\prime}\right)$.

This property is related to the Hahn-Banach theorem and norming sets.

Examples 1. The property $(\mathcal{P})$ satisfied in the following cases:

$(i)$ if $X$ is a normed space and $F$ contains the space $X^{*} . e:=\{x \mapsto p(x) . e$ : $\left.p \in X^{*}\right\}$, where $e \in Y$ is a fixed point such that $\|e\|=1$.

(ii) if $(X, d)$ is a metric space and $F$ contains the functions $d_{z}: x \mapsto$ $d(x, z) . e$, for all $z \in X$, where $e \in Y$ is a fixed point is such that $\|e\|=1$.

(iii) In particular, the $\operatorname{space}^{\operatorname{Lip}_{0}}(X, Y)$ satisfies the property $(\mathcal{P})$. If moreover, $X$ is a normed space, then $L(X, Y)$ has the property $(\mathcal{P})$ too.

Proof. ( $i$ ) By the Hahn-Banach theorem, for all $x \in X$ there exists $x^{*} \in X^{*}$ such that $\left\|x^{*}\right\|=1$ and $x^{*}(x)=\|x\|$. Then, for each $x \in X$, we consider the continuous linear map $p_{x}=x^{*} . e: X \rightarrow Y$ defined by $p_{x}(z)=x^{*}(z) e$ for all $z \in X$, and the property $(\mathcal{P})$ is satisfied.

(ii) Immediat.

(iii) This part follows from $(i)$ and (ii) respectively.

\section{The MAIN RESUlt}

We are going to give the proof of the main result of this note. Let $(X, d)$ be a metric space with a base point 0 and $Y$ be a Banach space. Let $F \subset$ $\operatorname{Lip}_{0}(X, Y)$ and $\phi: X \times X \rightarrow \mathbb{R}^{+}$be a positive function such that $\phi\left(x, x^{\prime}\right)=0$ if and only if $x=x^{\prime}$. For each real number $s>0$, we denote:

$$
\begin{gathered}
\mathcal{N}_{\phi, s}(F):=\left\{f \in F: \sup _{x, x^{\prime} \in X ; x \neq x^{\prime}} \frac{\left\|f(x)-f\left(x^{\prime}\right)\right\|}{\phi\left(x, x^{\prime}\right)} \leq s\right\}, \\
\mathcal{N}_{\phi}(F):=\left\{f \in F: \sup _{x, x^{\prime} \in X ; x \neq x^{\prime}} \frac{\left\|f(x)-f\left(x^{\prime}\right)\right\|}{\phi\left(x, x^{\prime}\right)}<+\infty\right\} .
\end{gathered}
$$


Notice that $\mathcal{N}_{\phi}(F)=\cup_{k \in \mathbb{N}} \mathcal{N}_{\phi, k}(F)$ and $\mathcal{N}_{\psi}(F) \subset \mathcal{N}_{\phi}(F)$ if $\psi \leq \phi$.

Theorem 1. Let $F$ be a nonempty (closed) convex cone of $\operatorname{Lip}_{0}(X, Y)$ satisfying $(\mathcal{P})$. Let $\phi: X \times X \rightarrow \mathbb{R}^{+}$be any positive function such that $\phi\left(x, x^{\prime}\right)=0$ if and only if $x=x^{\prime}$. Suppose that $\inf \left\{\frac{\phi\left(x, x^{\prime}\right)}{d\left(x, x^{\prime}\right)}: x, x^{\prime} \in X ; x \neq x^{\prime}\right\}=0$, then for every positive real number $s>0$, we have that $\mathcal{N}_{\phi, s}(F)$ is a porous subset of $\left(F,\|\cdot\|_{L}\right)$. Consequently, the following assertions are equivalent.

(1) $\mathcal{N}_{\phi}(F) \neq F$.

(2) $\inf \left\{\frac{\phi\left(x, x^{\prime}\right)}{d\left(x, x^{\prime}\right)}: x, x^{\prime} \in X ; x \neq x^{\prime}\right\}=0$.

(3) $\mathcal{N}_{\phi}(F)$ is a $\sigma$-porous subset of $\left(F,\|\cdot\|_{L}\right)$.

Proof. (1) $\Longrightarrow(2)$. Suppose that $\alpha:=\inf \left\{\frac{\phi\left(x, x^{\prime}\right)}{d\left(x, x^{\prime}\right)}: x, x^{\prime} \in X ; x \neq x^{\prime}\right\}>0$, then $\phi\left(x, x^{\prime}\right) \geq \alpha d\left(x, x^{\prime}\right)$ for all $x, x^{\prime} \in X$. It follows that for every $f \in F$, we have that

$$
\sup _{x, x^{\prime} \in X ; x \neq x^{\prime}} \frac{\left\|f(x)-f\left(x^{\prime}\right)\right\|}{\phi\left(x, x^{\prime}\right)} \leq\|f\|_{L} \sup _{x, x^{\prime} \in X ; x \neq x^{\prime}} \frac{d\left(x, x^{\prime}\right)}{\phi\left(x, x^{\prime}\right)} \leq \frac{\|f\|_{L}}{\alpha}<+\infty .
$$

Thus, $\mathcal{N}_{\phi}(F)=F$. Part $(3) \Longrightarrow(1)$ is trivial.

Let us prove that if $\inf \left\{\frac{\phi\left(x, x^{\prime}\right)}{d\left(x, x^{\prime}\right)}: x, x^{\prime} \in X ; x \neq x^{\prime}\right\}=0$, then for every $s>0$, we have that $\mathcal{N}_{\phi, s}(F)$ is a porous subset of $\left(F,\|\cdot\|_{L}\right)$, this gives in particular $(2) \Longrightarrow(3)$. Indeed, if $\inf \left\{\frac{\phi\left(x, x^{\prime}\right)}{d\left(x, x^{\prime}\right)}: x, x^{\prime} \in X ; x \neq x^{\prime}\right\}=0$, then there exists a pair of sequences $\left(a_{n}\right),\left(b_{n}\right) \subset X$ such that $0<r_{n}:=\frac{\phi\left(a_{n}, b_{n}\right)}{d\left(a_{n}, b_{n}\right)} \rightarrow 0$. By assumption, there exists $K_{F}>0$ and a sequence $\left(p_{n}\right) \subset F$ such that $\left\|p_{n}\right\|_{L} \leq K_{F}$ and $\left\|p_{n}\left(a_{n}\right)-p\left(b_{n}\right)\right\|=d\left(a_{n}, b_{n}\right)$, for all $n \in \mathbb{N}$. Let $f \in \mathcal{N}_{\phi, s}(F)$, then we have that $\sup _{x, x^{\prime} \in X ; x \neq x^{\prime}} \frac{\left\|f(x)-f\left(x^{\prime}\right)\right\|}{\phi\left(x, x^{\prime}\right)} \leq s$. It follows that

$$
\begin{aligned}
\frac{\left\|\left(f+\sqrt{r_{n}} p_{n}\right)\left(a_{n}\right)-\left(f+\sqrt{r_{n}} p_{n}\right)\left(b_{n}\right)\right\|}{\phi\left(a_{n}, b_{n}\right)} & \geq \sqrt{r_{n}} \frac{\left\|p_{n}\left(a_{n}\right)-p_{n}\left(b_{n}\right)\right\|}{\phi\left(a_{n}, b_{n}\right)}-\frac{\left\|-\left(f\left(a_{n}\right)-f\left(b_{n}\right)\right)\right\|}{\phi\left(a_{n}, b_{n}\right)} \\
& \geq \sqrt{r_{n}} \frac{d\left(a_{n}, b_{n}\right)}{\phi\left(a_{n}, b_{n}\right)}-\sup _{x, x^{\prime} \in X ; x \neq x^{\prime}} \frac{\left\|f(x)-f\left(x^{\prime}\right)\right\|}{\phi\left(x, x^{\prime}\right)} \\
& \geq \frac{1}{\sqrt{r_{n}}}-s
\end{aligned}
$$

Since, $r_{n} \rightarrow 0$, when $n \rightarrow+\infty$, there exists a subsequence $\left(r_{n_{m}}\right)$ such that

$$
\frac{1}{\sqrt{r_{n_{m}}}}>4 s, \quad \forall m \in \mathbb{N} \text {. }
$$

We set $f_{m}=f+\sqrt{r_{n_{m}}} p_{n_{m}} \in F$, for all $m \in \mathbb{N}$. We have that

$$
\left\|f_{m}-f\right\|_{L}=\sqrt{r_{n_{m}}}\left\|p_{n_{m}}\right\|_{L} \leq K_{F} \sqrt{r_{n_{m}}} \rightarrow 0 \text { when } m \rightarrow+\infty .
$$


Let us prove that $B\left(f_{m}, \frac{1}{2 K_{F}}\left\|f_{m}-f\right\|_{L}\right) \subset F \backslash \mathcal{N}_{\phi, s}(F)$ for all $m \in \mathbb{N}$. Indeed, let $g \in B\left(f_{m}, \frac{1}{2}\left\|f_{m}-f\right\|_{L}\right)$, then we have using the above informations that

$$
\begin{aligned}
\frac{\left\|g\left(a_{n_{m}}\right)-g\left(b_{n_{m}}\right)\right\|}{\phi\left(a_{n_{m}}, b_{n_{m}}\right)} & \geq \frac{\left\|f_{m}\left(a_{n_{m}}\right)-f_{m}\left(b_{n_{m}}\right)\right\|}{\phi\left(a_{n_{m}}, b_{n_{m}}\right)}-\frac{\left\|\left(f_{m}-g\right)\left(a_{n_{m}}\right)-\left(f_{m}-g\right)\left(b_{n_{m}}\right)\right\|}{\phi\left(a_{n_{m}}, b_{n_{m}}\right)} \\
& \geq\left(\frac{1}{\sqrt{r_{n_{m}}}}-s\right)-\left\|f_{m}-g\right\|_{L} \frac{d\left(a_{n_{m}}, b_{n_{m}}\right)}{\phi\left(a_{n_{m}}, b_{n_{m}}\right)} \\
& \geq\left(\frac{1}{\sqrt{r_{n_{m}}}}-s\right)-\frac{1}{2 K_{F}}\left\|f_{m}-f\right\|_{L} \frac{d\left(a_{n_{m}}, b_{n_{m}}\right)}{\phi\left(a_{n_{m}}, b_{n_{m}}\right)} \\
& \geq\left(\frac{1}{\sqrt{r_{n_{m}}}}-s\right)-\frac{1}{2} \sqrt{r_{n_{m}}} \frac{1}{r_{n_{m}}} \\
& =\frac{1}{2 \sqrt{r_{n_{m}}}}-s \\
& >s .
\end{aligned}
$$

Thus, we have that $g \in F \backslash \mathcal{N}_{\phi, s}(F)$ and so that $B\left(f_{m}, \frac{1}{2}\left\|f_{m}-f\right\|_{L}\right) \subset$ $F \backslash \mathcal{N}_{\phi, s}(F)$ for all $m \in \mathbb{N}$. Thus, $\mathcal{N}_{\phi, s}(F)$ is porous in $F$ (with $c=\frac{1}{2 K_{F}}$ ). It follows that $\mathcal{N}_{\phi}(F)=\cup_{k \in \mathbb{N}} \mathcal{N}_{\phi, k}(F)$ is $\sigma$-porous in $\left(F,\|\cdot\|_{L}\right)$.

2.1. Immediate consequences. We deduce immediately the result mentioned in the abstract.

Corollary 1. Let $X_{1}:=\left(X, d_{1}\right)$ and $X_{2}:=\left(X, d_{2}\right)$ be a set equipped with two metrics such that $d_{1} \leq d_{2}$ and let $(Y,\|\cdot\|)$ be a Banach space. Then, $\operatorname{Lip}_{0}\left(X_{1}, Y\right)$ is a $\sigma$-porous subset of $\operatorname{Lip}_{0}\left(X_{2}, Y\right)$ if (and only if) $d_{1}$ and $d_{2}$ are not equivalent, if and only if $\operatorname{Lip}_{0}\left(X_{1}, Y\right) \neq \operatorname{Lip}_{0}\left(X_{2}, Y\right)$.

Proof. We use Theorem 1 and part (iii) of Exemple 1 observing the following equality $\operatorname{Lip}_{0}\left(X_{1}, Y\right)=\mathcal{N}_{d_{1}}\left(\operatorname{Lip}_{0}\left(X_{2}, Y\right)\right)$.

Notice that if $0<\alpha \leq 1$ and $d$ is a metric, so is $d^{\alpha}$, hence the above corollay applies to the space of $\alpha$-Hölder-functions that vanish at $0_{X}$ which is $\operatorname{Lip}_{0}^{\alpha}(X, Y):=\operatorname{Lip}_{0}\left(X_{d^{\alpha}}, Y\right)$. Notice also that if $0<\alpha<\beta \leq 1$ and $d$ is bounded, then $\operatorname{Lip}_{0}^{\beta}(X, Y) \subset \operatorname{Lip}_{0}^{\alpha}(X, Y)$. The metrics $d^{\alpha}$ and $d^{\beta}$ are not equivalent if and only if, $\inf \left\{\frac{d^{\beta}\left(x, x^{\prime}\right)}{d^{\alpha}\left(x, x^{\prime}\right)}: x, x^{\prime} \in X ; x \neq x^{\prime}\right\}=0$, if and only if $\inf \left\{d\left(x, x^{\prime}\right): x, x^{\prime} \in X ; x \neq x^{\prime}\right\}=0$ (since $\beta>\alpha$ ). Thus, we get the result of the abstract.

Corollary 2. Let $(X, d)$ be a bounded metric space with a base point $0_{X}$, $(Y,\|\cdot\|)$ be a Banach space and $0<\alpha<\beta \leq 1$. Then, $\operatorname{Lip}_{0}^{\beta}(X, Y)$ is a $\sigma$-porous subset of $\operatorname{Lip}_{0}^{\alpha}(X, Y)$, if and only if $\inf \left\{d\left(x, x^{\prime}\right): x, x^{\prime} \in X ; x \neq x^{\prime}\right\}=0$.

For the linear case, we have the following results. 
Corollary 3. Let $X_{1}:=\left(X,\|\cdot\|_{1}\right)$ and $X_{2}:=\left(X,\|\cdot\|_{2}\right)$ be a linear space equipped with two norms such that $\|\cdot\|_{1} \leq\|\cdot\|_{2}$ and let $(Y,\|\cdot\|)$ be a Banach space. Then, $L\left(X_{1}, Y\right)$ is a $\sigma$-porous subset of $L\left(X_{2}, Y\right)$ if and only if $\|\cdot\|_{1}$ and $\|\cdot\|_{2}$ are not equivalent if and only if $L\left(X_{1}, Y\right) \neq L\left(X_{2}, Y\right)$.

Proof. We use Theorem 1 and part (iii) of Exemple 1 after observing that $L\left(X_{1}, Y\right)=\mathcal{N}_{\|\cdot\|_{1}}\left(L\left(X_{2}, Y\right)\right)$.

Example 1. Let $i:\left(l^{1}(\mathbb{N}),\|\cdot\|_{1}\right) \rightarrow\left(l^{1}(\mathbb{N}),\|\cdot\|_{\infty}\right)$ be the continuous identity map. Then the image of the adjoint $i^{*}$ of $i$ is a $\sigma$-porous subset of $\left(l^{\infty}(\mathbb{N}), \|\right.$. $\left.\|_{\infty}\right)$.

We give in the following corollary a connexion between the surjectivity of the adjoint $T^{*}$ of a one-to-one bounded linear operator $T$ and the non$\sigma$-porosity of its image (see in this sprit, the open mapping theorem in $[6$, Theorem 2.11]).

Proposition 1. Let $\left(X,\|\cdot\|_{X}\right)$ and $\left(Z,\|\cdot\|_{Z}\right)$ be Banach spaces. Let $T$ : $X \rightarrow Z$ be a one-to-one bounded linear operator and $T^{*}$ its adjoint. Then, the following assertions are equivalent.

(i) $T^{*}\left(Z^{*}\right)$ is not contained in a $\sigma$-porous subset of $X^{*}$.

(ii) There exists $\alpha>0$ such that $\alpha\|x\|_{X} \leq\|T(x)\|_{Z}$ for all $x \in X$.

(iii) $T^{*}$ is onto.

Proof. Since $T: X \rightarrow Z$ is a one-to-one bounded linear operator, then, the following map define another norm on $X$ :

$$
\|x\|:=\frac{\|T(x)\|_{Z}}{\|T\|} \leq\|x\|_{X}, \quad \forall x \in X .
$$

Let us denote $X_{1}:=(X,\|\cdot\|)$. By Corollary 3, applied with $Y=\mathbb{R}$, we have that $X_{1}^{*}$ is a $\sigma$-porous subset of $X^{*}$ if and only if $\|\cdot\|$ and $\|\cdot\|_{X}$ are not equivalente. Thus, if $(i i)$ is not satisfied (that is, $\|\cdot\|$ and $\|\cdot\|_{X}$ are not equivalente) then, since $T^{*}\left(Z^{*}\right) \subset X_{1}^{*}$ we get that $T^{*}\left(Z^{*}\right)$ is contained in a $\sigma$-porous subset of $X^{*}$. Hence, $(i) \Longrightarrow(i i)$ is proved. Now, suppose that (ii) holds, it follows that $T(X)$ is closed in $Y$. Let $x^{*} \in X^{*}$ and define $\phi$ on $T(X)$ by $\phi(T(x)):=x^{*}(x)$ for all $x \in X$. Clearly $\phi$ is well defined (since $T$ is one-to-one) and linear continuous on $T(X)$. Thus, $\phi$ extends to a linear continuous functional $y^{*} \in Y^{*}$ and we have $T^{*}\left(y^{*}\right)=y^{*} \circ T=x^{*}$. Hence, $T^{*}$ is onto and $(i i) \Longrightarrow($ iii $)$ is proved. Part $(i i i) \Longrightarrow(i)$, is trivial.

Let $(X,\|\cdot\|)$ be a normed space, and let $S$ be a nonempty subset of the dual space $X^{*}$. The set $S$ is called separating if: $x^{*}(x)=0$ for all $x^{*} \in S$ implies that $x=0$. It is called norming if the functional

$$
N_{S}(x)=\sup _{x^{*} \in S ; x^{*} \neq 0} \frac{\left|x^{*}(x)\right|}{\left\|x^{*}\right\|},
$$

is an equivalent norm on $X$ (see [2] for the use of this notion). 
Proposition 2. Let $(X,\|\cdot\|)$ be a normed space. Every separating subset $S \subset X^{*}$ which is not contained in a $\sigma$-porous subset of $X^{*}$, is norming.

Proof. It is clear that $N(x) \leq\|x\|$ for all $x \in X$. On the other hand, we have that

$$
S \subset \mathcal{N}_{N_{S}}(X):=\left\{x^{*} \in X^{*}: \sup _{N_{S}(x)=1}\left|x^{*}(x)\right|<+\infty\right\} .
$$

Since $S$ is not contained in a $\sigma$-porous subset of $X^{*}$, then $\mathcal{N}_{\phi}(X)$ must be non- $\sigma$-porous, which implies from Theorem 1 that $\inf _{\|x\|=1} N_{S}(x)>0$. Hence $N_{S}$ is equivalent to $\|\cdot\|$.

2.2. Application to the barrier cone and polar of sets. Let $X$ be a normed space and $K$ be a nonempty subset of $X$. The barrier cone of $K$ is the subset $B(K)$ of the topological dual $X^{*}$ defined by

$$
B(K)=\left\{x^{*} \in X^{*}: \sup _{x \in K} x^{*}(x)<+\infty\right\} .
$$

The polar set of $K$ is a subset of the barrier cone of $K$ defined as follows:

$$
K^{\circ}=\left\{x^{*} \in X^{*}: \sup _{x \in K} x^{*}(x) \leq 1\right\} .
$$

The study of barrier cones has interested several authors. It is shown in [4, Theorem 3.1.1] that for a closed convex subset $K$ of $X$, we have that $B(K)=X^{*}$ if and only if $K$ is bounded, on the other hand, $B(K)$ is dense in $X^{*}$ if and only if $K$ does not contain any halfline. In general, we know that $\overline{B(K)} \neq X^{*}$ (see example in [1]). A study of the closure of the barrier of a closed convex set is given in [1]. As an immediat consequence of main theorem, we obtain bellow that the barrier cone of some general class of unbounded subsets is a $\sigma$-pourous subset of the dual $X^{*}$. This shows that in general, the barrier cone may be a "very small" subset of $X^{*}$.

We define the classe $\Phi(X)$ of positive functions on $X$ (not necessarily continuous) as follows: $\phi \in \Phi(X)$ if and only if, $\phi: X \rightarrow \mathbb{R}^{+}$and satisfies

(i) $\phi(\lambda x)=|\lambda| \phi(x)$ for all $x \in X$ and all $\lambda \in \mathbb{R}$

(ii) $\phi(x)=0$ if and only if $x=0$

For every $\phi \in \Phi(X)$, we denote $S_{\phi}:=\{x \in X: \phi(x)=1\}$ and $C_{\phi}:=$ $\{x \in X: \phi(x) \leq 1\}$. Notice, that in general $C_{\phi}$ is not a convex set (resp. not closed), if we do not suppose that $\phi$ is a convex function (resp. a continuous function). It is easy to see that

(1) $C_{\phi}$ is bounded $\Longleftrightarrow S_{\phi}$ is bounded $\Longleftrightarrow \inf _{x \in X:\|x\|=1} \phi(x)>0$. 
Thanks to the symmetry of $\phi \in \Phi(X)$ and the fact that $B\left(C_{\phi}\right)=B\left(S_{\phi}\right)$, we have using the notation of Theorem 1 , that

$$
B\left(C_{\phi}\right)=\mathcal{N}_{\phi}\left(X^{*}\right) .
$$

The polar of $S_{\phi}$ coincides with $\mathcal{N}_{\phi, 1}\left(X^{*}\right)$ and we have

$$
C_{\phi}^{\circ} \subset S_{\phi}^{\circ}=\mathcal{N}_{\phi, 1}\left(X^{*}\right) \text {. }
$$

Now, using (1), (2) and (3) and applying Theorem 1 to the spaces $F=X^{*}$ and $Y=\mathbb{R}$ (using Exemple 1), we get directly the following informations about the size of the barrier cone, as well as the polar of a set of the form $C_{\phi}$ in the dual space.

Corollary 4. Let $X$ be a normed space and $\phi \in \Phi(X)$. If $C_{\phi}$ is not bounded in $X$, then the polar $C_{\phi}^{\circ}$ is contained in a porous subset of $X^{*}$. Moreover, the followin assertions are equivalent.

(i) $B\left(C_{\phi}\right) \neq X^{*}$.

(ii) $C_{\phi}$ is not bounded in $X$.

(iii) $B\left(C_{\phi}\right)$ is a $\sigma$-porous subset of $X^{*}$.

We deduce that, if $K$ is any nonempty subset of $X$ such that $S_{\phi} \subset K$, for some $\phi \in \Phi(X)$ with $S_{\phi}$ not bounded, then the polar $K^{\circ}$ is contained in a porous subset of $X^{*}$ and the barrier cone $B(K)$ is contained in a $\sigma$-porous subset of $X^{*}$. Notice that if $K$ is a closed absorbing disk in $X$, does not contain a non-trivial vector subspace and is a neighborhood of the origin in $X$, then the Minkowski functional $\phi_{K}$ of $K$ is a continuous norm (with respect to the norm $\|\cdot\|$, but not equivalent to it, if $K$ is not bounded) hence $\phi_{K} \in \Phi(X)$ and we have that $K=C_{\phi_{K}}$.

\section{REFERENCES}

1. S. Adly, E. Ernst, M. Théra Norm-closure of the barrier cone in normed linear spaces. Proc. Am. Math. Soc. 132 (10), (2004) 2911-2915.

2. G. Godefroy, N. J. Kalton, Lipschitz-free Banach spaces, Studia Math. 159 (2003), 121-141.

3. Gilles Godefroy, The use of norm attainment, Bull. Belg. Math. Soc. Simon Stevin 20 , no. 3 (2013), 417-423.

4. T. Laetsch Normal cones, barrier cones, and the spherical image of convex surfaces in locally convex spaces. Pacific J. Math. 73 (1) (1977), 107-123.

5. J. Lindenstrauss and D. Preiss On Fréchet differentiability of Lipschitz maps between Banach spaces, Ann. of Math.157 (2003), 257-288.

6. Rudin, Walter Functional Analysis. International Series in Pure and Applied Mathematics. 8 (1991) (Second ed.). New York.

7. Zajíček, L. Porosity and $\sigma$-porosity, Real Anal. Exchange. 13 (2) (1987-1988), 314-350.

Laboratoire Samm 4543, Université Paris 1 Panthéon-Sorbonne, Centre P.M.F. 90 Rue Tolbiac, 75634 Paris Cedex 13, France

Email address: Mohammed.Bachir@univ-paris1.fr 